\title{
Effects of Mobile Phone Radiation on Human Health
}

\section{Shazia Saqib}

\begin{abstract}
In past 20 years the use of mobile phone has increased manifold. After 2015 there are more mobiles than humans on this earth. This makes the world a hub of communication as well as Centre of social network.

There is a huge growth of antennas and towers to support increasing trend of mobile. Along with that, there is a growing concern that radiations emitted by Mobile Stations or these towers are dangerous for health. Although many believe that it is yet to be proved that these radiations are injurious to health, especially harmful to heart, brain, nervous system and many more, but a large number of mobile users know that mobile radiations are dangerous for sure. This research is an effort to find actual impacts of radiations by mobiles on human health and to find the impact of heavy mobile usage on our society as physical contact has been now replaced to peer to peer (p2p) on the phone.
\end{abstract}

Keywords-Ionization Energy, SAR, Electromagnetic Spectrum;

\section{INTRODUCTION}

In the past decade or two advancements in computing and communication shows the world is changing very fast. In closed protective environments mainframe systems has been replaced by modern computing devices e.g. laptops palmtops etc. legacy system faced immobility problem due to size and energy requirements.

The heterogeneity of the technology achieves the autonomy in accessing the information which enable accessing of information anywhere. The traditional solid state medium of networks has been replaced with wireless transmission medium which are the part of electromagnetic (EM) spectrum. This transformation of technology has taken society by storm [1].

As these technologies are spreading business, conflicting issues arise side by side. ${ }^{2}$

Independent research provide serious consequences about our living environment is highly




saturated with electromagnetic fields. The competent authorities confirm that observations are. unknown to them. All these devices including mobile phones, base station antennas on towers and buildings throughout the world emit radiofrequency (RF) radiation. The strength of these radio waves is higher in populated areas. The radiations are of two types: Ionizing Radiation and Non-Ionizing Radiation [1]

In the electromagnetic spectrum with short wave and high frequency, ionizing radiation is highest contributor. The exposure of ionizing radiation causes burns, hair loss, cancer, birth defects in infants, immediate death and other illnesses. The effect of Ionizing radiation for an atom is enough to pull an electron, thus creating an icon.

Although high level ionizing rays are dangerous for health, but low level exposure shows benefits in the medical field to make easier to diagnose problems. The 300 joules ionization radiation with short wave and high frequency is enough to kill human being. If we increase the wavelength and low the frequency of ionization the effect will much low like 1.5 million joules of radiation will be equal to 300 joules. High wavelength and low frequency ionization radiation includes infra-red light, microwaves, and radio frequency [1].

Specific Absorption Rate (SAR) measures the health effects of the non-ionizing radiation. Which defines, as the power absorbed per mass of tissue and has units of watts per kilogram (W/kg) [1]. There are many countries that set the SAR's maximum levels for modern handsets in many countries. FCC has set a SAR limit for USA of $1.6 \mathrm{~W} / \mathrm{kg}$, averaged over a volume of 1 gram of tissue of head and $2 \mathrm{~W} / \mathrm{Kg}$ over volume of 10 grams of tissue in Europe [2].

The base stations and the Cell Phone strength in an area determines the Radio Frequency measure.

- average talk time of cell phone in a month as little as 500 minutes can increase the probability of brain cancer by $140 \%$ to $300 \%$.

- Cell phone radiation damages living DNA.

- Cell phone radiation affects sensitive brain tissues.

- Six years use of cell phone increase the risk of developing auditory nerve tumor by $50 \%$

- Cell phone two-minute call affects a children alter brain function for an hour.

- Cordless phones are even higher cancer risks than cell phones [3]

Russians have used this technology to intercept conversation within the US embassy compound, result was the American embassy staff member was diagnosed with leukemia and sent back to USA. The person replacement got same disease too, Staff members of US Embassy also complained other problem to them such as memory loss, brain fog, loss of focus and insomnia during their services in the embassy [3].

These radiations are dangerous both for environment and for human. Another issue is getting disposed of old computing devices which contain plastic another health hazard. The plastics 
heavy metals, gasses create products that are dangerous for human beings. Electromagnetic pollution is the most dangerous form of pollution as it is invisible and insensible [3].

Besides the physical waste the other important concern due to increase of such devices is the energy consumption, which increase day by day. A huge number of waste produced are stored in attics, basements, office closets and storerooms. These computers are made of over a thousand materials, such as lead, cadmium and mercury. Some of these materials are known to be highly toxic.

Lead from these devices prevents us from dumping these devices. If it leaks into water systems, it can create havoc. Four to six-pound lead contains in a monitor [4], and the mixture of phosphorous with the lead protect user from radiation. The waste growth ratio of electronic equipment in three time more than other municipal waste [5].

In the recent development in wireless and PDA technology reduced the lifetime and cost of the devices. The devices changing with the user requirements with minor updates. Like one system doesn't has Bluetooth and the other has.

\section{FINDINGS}

All radiation is not harmful which invisibly move through our body. We know that invisible EMR waves can move easily through the concrete walls of buildings. These EMR can easily pass through our body soft tissue. Signal communication through barriers and obstruction connects from tower to tower.

Long-term EMR exposure risked and increased causes our society sicker, some founding's are listed

The high level sicknesses of EMR Exposure are Sleep disorders and insomnia, headaches and migraines, immune system disorders, increased blood pressure, Learning disabilities, heart disease, leukemia, fatigue memory loss, depression, Loss of concentration, DNA damage, Brain tumors, Alzheimer's disease, Parkinson's disease, Autism, hormonal imbalance etc. [3]

\subsubsection{Ethical and Social Impressions}

The survey shows that doctors and psychologists agree that mobile phone is a hazard both to health and social values.

Mobile communication makes people introverted, isolated and increase inability to deal in face to face communication and interaction. [6] International Agency for Research on Cancer (IARC), report by World Health Organization. WHO published in USA today, medical experts say that cell phones are "possible carcinogens". Although there isn't much evidence. We're not going to see the effects of the heavier users for a decade or so. [7] [8]

Global System for Mobile Communication (GSM) and Universal Mobile Telecommunication System (UMTS) phones user are considered in this study. Literature shows that UMTS phone power is lower than the GSM phone in the average use. We see the increase in the use of mobile phone with the advancement in the technology but the other factor of time with the exposure of the brain to radio radiation is decreased. [9] 
The duration of minimum ten years casus the increased risk in developing brain tumor of ten years. Various epidemiological studies show links on long-term use of mobile phones and s increase in brain tumor risk [10]. The number of indirect health hazards connected to mobile phones which are caused just to use of it. Phone use while driving vehicle is observed unusually which cased road accident, is the best example of it.

Austria, France, and Germany national radiation advisory authorities recommended to their citizens measures to minimize exposure. Use of hands-free to keep mobile away from the head to decrease the radiation and to keep the mobile phone away from the body. [2]

EEA in September 2007 recommended some awareness about the risks of mobile phone uses, citizens should be familiar with this report. The sufficient evidence in EEA of risk to society and especially children. Mobile phone should not place in their heads: text messaging etc. Handsfree kits reduce the radiation levels to about ten times, then when the phone is pressed to the head.

\section{CONCLUSION}

Governments should label mobile handsets as a 'possible carcinogen', in line with the IARC decision. Our society needs to sit and chalk out rules and ethics for mobile communication. Perhaps like smoking, they need to put a warning may be on mobiles or may be at the start of every call, that too much use of this device is dangerous for health as well as for mental capabilities [11]. This can be concluded that the exposure of EMF radiation cases due to heavy cellphones caution is needed using the cell phones. More research work and development is needed for risk assessment based on higher number of long-term users. [12]. There is a dire need to setup bodies in collaboration with health department to conduct research at serious level. 
Volume No. 1, Issue No. 1 (Jan-March 2017) pp.37-43

\section{REFERENCES}

[1]: Health risks from mobile phone radiation - why the experts disagree. (2011, OCT 12). Retrieved from www.eea.europa.eu/ News / Health risks from mobile phone radiation - why the experts disagree.

[2]: The Cell Phone Poisoning of America. (2008). Logical Health LLC.

[3]: Mobile Phone Use and Brain Tumors in Children and Adolescents: A Multicenter CaseControl Study. (2011). JNCI.

[4]: A. Raouf Khan, N. Z. (2008). Health Hazards Linked to Using Mobile Cellular Phones. Journal of Information \& Communication Technology, 101-108.

[5]: Ashraf A. Aly, S. B. (2008). Research Review on the Biological Effect of Cell Phone Radiation on Human. IEEE.

[6]: Cooper, C. (2001, Sepetember 10). Where do old computers go to die? Retrieved from CNETNews.com.

[7]: FranzAdlkofer, I. B. (2009, March). How Susceptible Are Genes to Mobile Phone Radiation? State of the Research-Endorsements of Safety and Controversies-Self-Help Recommendations.

[8]: King, R. (2011, june 3). what-the-whos-cellphone-cancer-statement-really-means.htm. Retrieved from www.ieee.org.

[9]: Mary Brophy Marcus, L. S. (2011, June 1). WHO Cellphones possibly carcinogenic USATODAY.com. Retrieved from www.usatoday.org.

[10]: Ravi Jain, J. W. (2002). Environmental Design for Pervasive Computing Systems. MOBICOM' 02, 23-28.

[11]: Savita Chauhan, D. o. (n.d.). ENVIRONMENTAL AND HEALTH HAZARDS OF MOBILE DEVICES.

[12]: Yilong Lu, Y. H. (2012). Biological Effects of Mobile Phone Radiation. IEEE. 